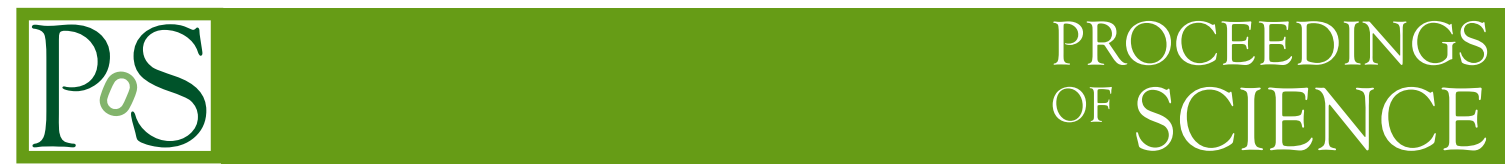

\title{
Jet production in diffractive processes at HERA
}

\author{
Toshinari Tawara* ${ }^{\dagger}$ \\ University of Tokyo, Japan \\ E-mail: tawara@mail.desy.de
}

\begin{abstract}
Dijet production in diffractive deep inelastic scattering (DIS) and photoproduction has been measured with both $\mathrm{H} 1$ and ZEUS detectors at HERA. The cross section for diffractive dijets in DIS is sensitive to the gluon contribution in the diffractive reaction. The results are compared to next-toleading order (NLO) QCD predictions using various diffractive parton density functions (DPDFs) obtained from the measurements of $F_{2}^{D}$. In the case of photoproduction, the cross sections are well described in shape by the NLO prediction using a DPDF which describes the cross section in DIS. But, the magnitude is about half of the measured cross sections. The results indicate a global suppression in diffractive photoproduction, although the uncertainty in the DPDFs needs to be evaluated before firmer conclusions can be made.
\end{abstract}

International Europhysics Conference on High Energy Physics

July 21st - 27th 2005

Lisboa, Portugal

\footnotetext{
* Speaker.

$\dagger$ On behalf of the H1 and ZEUS Collaborations
} 


\section{Introduction}

The cross section of deep inelastic scattering (DIS) of a proton and a lepton is described with a convolution of universal parton density functions of the proton and hard scattering cross sections between the partons and the lepton. As it was proven that this can also be applied in diffractive DIS [1], the diffractive cross section of DIS factorizes into diffractive parton density functions (DPDFs) and the hard scattering. Various sets of DPDFs have been obtained from DGLAP QCD fits to inclusive diffractive cross sections, in which the gluon DPDF is obtained from scaling violations. In contrast, diffractive dijet production is directly sensitive to the diffractive gluon density.

On the other hand, it is known that the diffractive cross sections for dijet processes in $p \bar{p}$ collisions at Tevatron is significantly (factor 3-10) lower than next-to-leading order (NLO) QCD prediction using the DPDFs obtained at HERA [2]. The suppression has been explained by rescattering between the proton remnant and the scattered proton. The diffractive dijet process in resolved photoproduction at HERA is similar to $p \bar{p}$ collisions at Tevatron. In resolved processes, the photon can be regarded as a hadron-like object containing many partons, while direct processes can be regarded as an interaction of a point-like photon. The two processes can be separated by using a measurable variable $x_{\gamma}^{\text {obs }}$, which corresponds to the longitudinal momentum fraction of the virtual photon entering in the hard subprocess. Resolved photon processes correspond to $x_{\gamma}^{\text {obs }}<1$, and direct photon processes to $x_{\gamma}^{\mathrm{obs}} \simeq 1$. Therefore, diffractive dijet photoproduction is considered to be an ideal testing ground for rescattering between the proton remnant and the photon remnant. The suppression of diffractive dijet process in photoproduction and DIS is studied at HERA.

\section{Diffractive dijets in photoproduction and DIS}

Fig.1 shows the $\mathrm{H} 1$ results on the ratio of data to NLO predictions of the diffractive dijet production for photoproduction $\left(Q^{2}<0.01 \mathrm{GeV}^{2}\right)$ and DIS $\left(Q^{2}>4 \mathrm{GeV}^{2}\right)$ as a function of $y$ [3], where $Q^{2}$ is the photon virtuality and $y$ is the energy fraction of the electron carried by the exchanged photon. Both measurements are done in a common kinematic range; $165<W<242$ $\mathrm{GeV}, x_{I P}<0.03, E_{T}^{* \text { jet1 }}>5 \mathrm{GeV}, E_{T}^{* \text {,jet2 }}>4 \mathrm{GeV}$ and $-3.0<\eta_{\text {jets }}^{*}<0$, where $W$ is the photonproton centre-of-mass energy, $x_{\mathbb{P}}$ is the proton longitudinal momentum fraction of the diffractive exchange, $E_{T}^{* \text { jet } 1}\left(E_{T}^{* \text {,jet2}}\right)$ is the transverse energy of the first (second) highest $E_{T}$ jet and $\eta_{\text {jets }}^{*}$ is the pseudorapidity of the jets reconstructed in the photon-proton centre-of-mass frame ( $\gamma^{*} p$ frame). The jets are reconstructed using the inclusive $k_{T}$ algorithm in the $\gamma^{*} p$ frame. The NLO prediction is made with the H1 2002 fit (prel.) DPDFs [4]. The cross section in DIS is well described by the NLO prediction, while photoproduction is suppressed by a factor of about 0.5 .

ZEUS observes a similar suppression in photoproduction [5]. Fig.2 shows the cross section as a function of $x_{\gamma}^{\text {obs }}$. The kinematic range is $E_{T}^{\mathrm{jet} 1}>7.5 \mathrm{GeV}, E_{T}^{\mathrm{jet} 2}>6.5 \mathrm{GeV},-1.5<\eta_{\text {jets }}<1.5$, and $0.2<y<0.85$, where the jet variables are defined in the laboratory frame. It shows that the suppression is present in both high and low $x_{\gamma}^{\text {obs }}$ region. This is in conflict with the simple expectation that only the resolved process is suppressed by rescattering effects. A similar conclusion was drawn from the $\mathrm{H} 1$ measurements. 


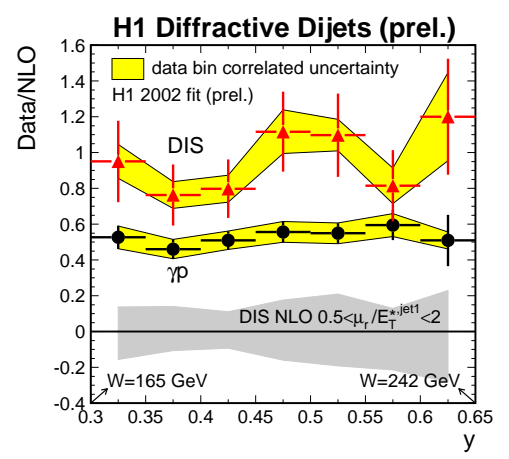

Figure 1: Ratio of the dijet data (See text) to NLO QCD predictions based on the H1 2002 fit (prel.) DPDFs for photoproduction and DIS in $y$. The vertical error bars indicate the uncorrelated experimental uncertainties. The error band around the ratio points shows an additional experimental normalization uncertainty. The NLO predictions are corrected for hadronization effects.

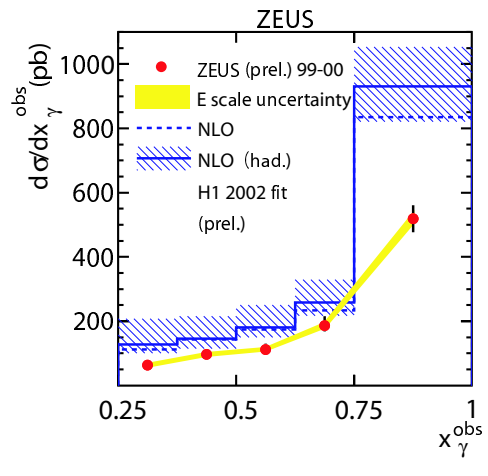

Figure 2: The dijet cross sections in photoproduction (See text) in $x_{\gamma}^{\text {obs }}$ compared to NLO predictions based on H1 2002 fit (prel.) DPDFs for photoproduction. The data are shown as dots. The shaded band represents the energy scale uncertainties. The dashed lines are NLO prediction. The solid lines are NLO predictions corrected for hadronization effects. The hatched area is renormalization scale uncertainties.

\section{Comparison to NLO predictions with various DPDFs}

New measurements of diffractive DIS in ZEUS [6] have been made in the kinematic range $5<$ $Q^{2}<100 \mathrm{GeV}^{2}, 100<W<250 \mathrm{GeV}, E_{T}^{* \text { jet } 1}>5 \mathrm{GeV}, E_{T}^{* \text {,jet2 }}>4 \mathrm{GeV}$ and $-3.5<\eta_{\text {jets }}^{*}<0$. Fig. 3 shows the diffractive dijet cross section in DIS as a function of $Q^{2}$ and $z_{\mathbb{P}}^{\text {obs }}$, where $z_{\mathbb{P}}^{\text {obs }}$ corresponds to the longitudinal momentum fraction of the parton to the hard scattering. NLO predictions are also shown using three sets of DPDFs: the H1 2002 fit; the ZEUS-LPS fit [7], obtained from the diffractive events with ZEUS Leading Proton Spectrometer and the charm contribution to $F_{2}^{D}$ [8]; and the GLP fit [9] using the ZEUS $F_{2}^{D}$ data with the $M_{X}$ method [10]. The latter two use the same theoretical framework. The data is well described by the prediction with the ZEUS-LPS fit and the H1 2002 fit, consistent with the conclusions of the H1 analysis. The NLO prediction with the GLP DPDFs is below the data. The differences of the shape in $z_{\mathbb{P}}^{\text {obs }}$ between NLO predictions and data are also large. The dijet cross section in DIS will help to further constrain the diffractive gluon density if it is included in the QCD fits to the $F_{2}^{D}$ data.

\section{Conclusion}

Diffractive dijet cross sections are measured in photoproduction and DIS with the $\mathrm{H} 1$ and ZEUS detectors. The cross sections are compared with NLO QCD predictions with DPDFs extracted from DGLAP QCD fits to HERA $F_{2}^{D}$ measurements. The NLO predictions with various DPDFs show large variation in dijet cross sections in DIS, reflecting large uncertainties in DPDFs. The measured dijet cross sections will give extra constraints to determine the DPDF. Diffractive dijet cross sections in photoproduction are lower by a factor of about 0.5 than the expectation, when using a particular DPDF. However, the NLO prediction with one set of DPDFs might not simultaneously describe photoproduction and DIS for diffractive dijet data. There is no indication 


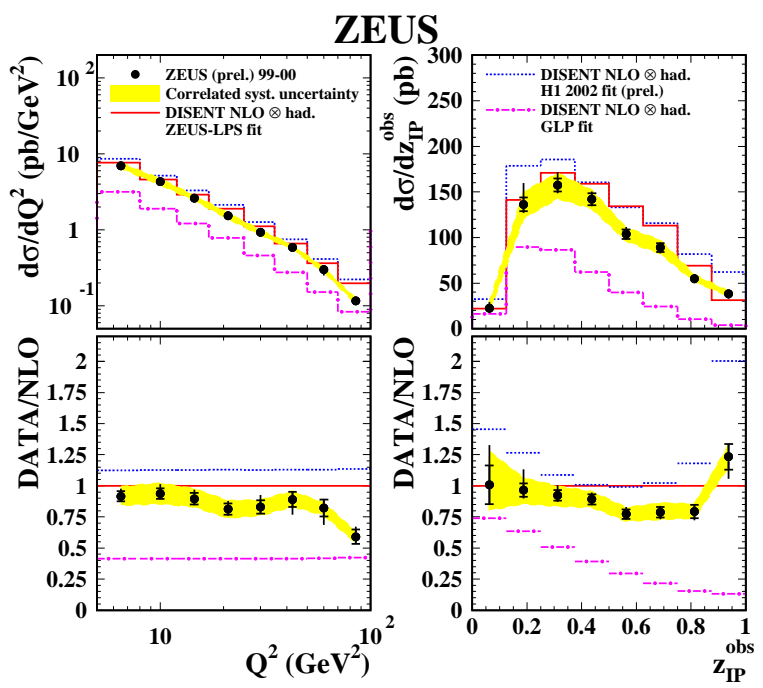

Figure 3: Comparisons of the dijet cross sections in DIS by ZEUS (See text) between data and NLO predictions in $Q^{2}$ and $z_{\mathbb{P}}^{\mathrm{obs}}$. The upper plots show the cross sections compared to the three NLO predictions, while the lower plots show the ratio of the cross sections over NLO predictions from ZEUS-LPS fit. The data are shown as dots. The inner error bars represent the statistical uncertainty, while the outer error bars represent the statistical and systematic uncertainties added in quadrature. The shaded band represents the correlated error, which is the sum of the energy scale and proton dissociation background uncertainties. The solid lines represent NLO cross sections from ZEUS-LPS fit, while NLO predictions from H1 2002 fit (prel.) are drawn with a dotted line. The predictions based on GLP fit are shown as a dash-dotted line.

in dijet photoproduction of a different suppression for the resolved and direct components. We need to further understand the partonic structure of the diffractive exchange.

\section{References}

[1] J. Collins, Phys. Rev. D 573051 (1998); J. Collins, J. Phys. G 281069 (2002).

[2] CDF Collaboration, T.Affolder et al., Phys. Rev. Lett. 845043 (2000).

[3] H1 Collaboration, Dijets in Diffractive Photoproduction and Deep-Inelastic Scattering at HERA, PoS(HEP2005)634.

[4] H1 Collaboration, paper 980 submitted to 31st Intl. Conf. on High Energy Physics, ICHEP 2002, Amsterdam.

[5] ZEUS Collaboration, Diffractive photoproduction of dijets at HERA, PoS(HEP2005)338.

[6] ZEUS Collaboration, Dijet production in diffractive deep inelastic scattering at HERA, PoS(HEP2005)342.

[7] ZEUS Collaboration, Eur. Phys. J. C 38, 43 (2004).

[8] ZEUS Collaboration., Nucl. Phys. B 672, 3 (2003).

[9] M. Groys, A. Levy and A. Proskuryakov, to be published in the Proc. of the HERA-LHC Workshop, DESY/CERN (2005).

[10] ZEUS Collaboration, accepted by Nucl. Phys. B, DESY 05-011 (2005). 